\title{
How Do The Number of Missing Daily Diary Days Impact The Psychometric Properties And Study Results Arising From a Weekly Average Summary Score?
}

\author{
Pip Griffiths ( $\sim$ pip.griffiths@iqvia.com ) \\ IQVIA https://orcid.org/0000-0002-1031-5802 \\ Abi Williams \\ IQVIA \\ Elaine Brohan \\ World Health Organization Regional Office for Europe
}

Research Article

Keywords: Daily diary data, missing data, summary score, psychometric properties, 4/7 days

Posted Date: November 8th, 2021

DOI: https://doi.org/10.21203/rs.3.rs-799794/v1

License: (c) (i) This work is licensed under a Creative Commons Attribution 4.0 International License. Read Full License 


\section{Abstract}

\section{Purpose}

Quality of life research often collects daily information and averages this over a week, producing a summary score. When data are missing, arbitrary rules (such as requiring at least $4 / 7$ observations) are used to determine whether a patient's summary score is created or set to missing. This simulation work aimed to assess the impact of missing data on the estimates produced by summary scores, the psychometric properties of the resulting summary score estimates and the impact on efficacy results.

\section{Methods}

Complete longitudinal data was simulated for 1000 samples of 400 patients with different day-to-day variability. Data was deleted from these samples in line with missingness mechanisms to create scenarios with up to six days of missing data. Summary scores were created for complete and missing data scenarios. Summary score estimates, psychometric properties and the ability to detect treatment differences were assessed for missing data scenarios compared to complete data.

\section{Results}

In most cases, the 4/7 day rule was supported, but this depended on daily variability. Fewer days of data were sometimes acceptable, but this was also dependent on the type of analyses conducted. An interactive tool was created to allow researchers to assess the impact of missing data in their own work.

\section{Conclusions}

This work suggests that the missing data rule used to create summary scores impacts on the estimate, measurement properties, interpretation and observed treatment effect. However, the way the summary score is derived does not have a uniform impact across these analyses.

\section{Introduction}

When a health condition can vary rapidly in a short space of time, assessment at periodic clinic visits may not be the most appropriate way of assessing change. This is a feature of many aspects of how a patient feels or functions, most notably in the assessment of mood, respiratory symptoms, pruritis and pain. A retrospective patient reported outcome (PRO) over a period where a health state has fluctuated may lead to an inaccurate representation of the construct being measured. Indeed, when comparing daily diary assessments to assessments completed at clinic visits in asthma, daily measurement has been shown to be more sensitive to detecting changes in patient health over time[1]. Additionally, retrospective reports of pain[2] and COPD[3] have been shown to be more likely to be inflated (i.e. worse symptom report) compared to daily report.

Daily measurement can now be conducted using electronic diaries, which can increase both the integrity and accuracy of the data collected[4] compared with paper completion. For example, prior to the invent of electronic data collection, where each entry is automatically time-stamped for assurance of integrity[4], research showed that participants tended to complete the diary questionnaire retrospectively by filling out many daily assessments at one timepoint[5]; a phenomenon known as the "parking lot effect". This leads to what appears to be a high completion rate, but is based on retrospective rather than daily completion. This may result in potentially inaccurate data due to recall effects. Electronic data collection can, on the other hand, prevent the completion of daily diaries outside of an allotted time window, set by the investigator. This allows for data integrity to be preserved but can lead to missing data when participants do not, for some reason, complete the diary in the allotted time[6]. Although good electronic daily diary compliance rates ( $>90 \%$ ) have typically been reported [5,7], accounting for missing data is still an important consideration with this mode of administration. Research has shown that PRO measure completion can vary, and specifically decline, over the course of a trial, potentially due to increased technical or condition related issues in the completing the diary the longer patients are enrolled[8]. High completion should therefore not be taken for granted. Missing data in daily diary studies can occur for a broad range of reasons including forgetting, being too busy, technical malfunction of the device, being too unwell to complete the diary[9] or simply not wanting to complete the questionnaire each day. The level of burden placed on participants is also an important consideration, particularly for data that is event-driven rather than daily and may require multiple completions over a day e.g. seizure data. Due to the variability inherent in event-driven diary data, this paper focuses on daily diary data though many of the considerations will also apply to event-driven diary data, where participants are required to complete the diary when a certain condition related event occurs (for example when suffering an episode of dysphagia). The reasons for missing daily diary assessment all link to statistical definitions of missing data that are often considered when analysing PRO results which contain missing records (Table 1).

\section{Table 1. Examples of Missingness Mechanisms and their consequences}




\begin{tabular}{|c|c|c|c|}
\hline $\begin{array}{l}\text { Missingness } \\
\text { mechanism }\end{array}$ & Definition & $\begin{array}{l}\text { Daily diary example (influences } \\
\text { missingness) }\end{array}$ & Issues \\
\hline $\begin{array}{l}\text { MCAR } \\
\text { (Missing } \\
\text { Completely at } \\
\text { Random) }\end{array}$ & $\begin{array}{l}\text { Missingness is unrelated to the construct being } \\
\text { observed. }\end{array}$ & $\begin{array}{l}\text { - Technical malfunction } \\
\text { - Translation not available } \\
\text { in participant's language } \\
\text { - Forgetting* }\end{array}$ & Increased variability \\
\hline $\begin{array}{l}\text { MAR } \\
\text { (Missing at } \\
\text { Random) }\end{array}$ & $\begin{array}{l}\text { After conditioning on observed data, missingness is not } \\
\text { related to the unobserved missing value. }\end{array}$ & 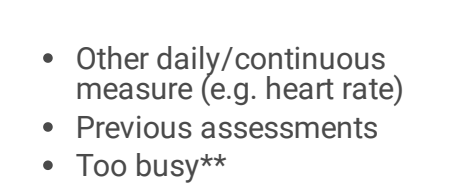 & $\begin{array}{l}\text { Increased variability } \\
\text { and potential bias }\end{array}$ \\
\hline $\begin{array}{l}\text { MNAR } \\
\text { (Missing Not at } \\
\text { Random) }\end{array}$ & $\begin{array}{l}\text { The missingness is directly related to the unobserved } \\
\text { missing value. }\end{array}$ & $\begin{array}{l}\text { - Too sick } \\
\text { - Too busy** }\end{array}$ & $\begin{array}{l}\text { Increased variability } \\
\text { and bias }\end{array}$ \\
\hline \multicolumn{4}{|c|}{ *Provided the construct under investigation is not memory } \\
\hline
\end{tabular}

Although these missing data mechanisms have been well documented in the literature, missing data still poses a challenge for statistical analysis[see 10]. These challenges mainly arise due to the difficulty in assigning missingness in a dataset to one of the categories presented in Table 1. The true underlying value of any unobserved missing data is by definition unknown, meaning it can be difficult to distinguish between missing data mechanisms (specifically MAR and MNAR) analytically. To account for this, it is recommended that multiple analysis approaches are used, each with different missing data assumptions, in order to assess the robustness of any results to the assumptions made[11]. Although these recommendations have been made for clinical trial analyses, similar considerations should also apply for psychometric analyses.

Missing data can lead to a reduction in the precision of a treatment effect and in the most severe cases can lead to biased estimates, especially if the reasons for the missing diary assessments are believed to be linked to the condition being assessed[12]. This is of particular concern when assessing data arising from daily diary collection due to the way the data is summarized in a trial setting. For example, to account for the daily variability in the condition of interest, daily assessment is often summarized over a time period such as a week by taking the mean of all assessments in that week[13]. This is a particularly typical way to assess diary data in clinical trial work where it is preferable to have a summary score which is linked to a participant's clinic visit. When daily diary data are missing, a common practice rule of thumb is to average over the remaining data in the time period, providing that participants have completed at least the majority of diary days in that time period (e.g. 4 or 5 days out of 7). Participants with fewer days of data than this cut off have a missing data value for the summary score, and are often not included in subsequent analyses.

Until recently, there was little evidence for this rule of thumb and the choice of averaging participant data provided that 4 or 5 days of data were present was mainly based on the researcher's prior experience. Initial simulation work in this area showed that group level estimates which were based on a summary score created when patients had at least three days of daily diary data were closer to the true, simulated, score in terms of variability than when the patients with missing data were excluded from the estimate[14]. In addition, reliability of the summary score over time was more likely to be preserved when summary scores included participants with as few as 3 days of diary data, rather than excluding participants who have missing data[15, Floden et al., in preparation].

Given that the rule of thumb currently employed to create summary scores from missing data over a week may not be the optimal strategy when it comes to the accuracy of the weekly summary score estimate or its reliability over time, it is important to understand whether other properties of such summary scores are better served by using all available data, or by excluding participants who do not have complete data. This is a particularly pertinent issue for clinical trial work where the reliability of the summary score, the validity of the summary score (i.e. how accurately it assesses the construct of interest [16]), the ability of the summary score to detect change and the interpretation of that score are crucial to properly assessing the efficacy of a potential new investigational medical product.

This paper uses a simulation approach to test these questions across different types of missing data and across scenarios which represented diseases with low, moderate and high daily variability. The following hypotheses will be specifically tested:

1. How many days of daily diary data are needed to create an accurate score for different missingness mechanisms, different variabilities of daily scores and different proportions of patients with missing data

Page $3 / 10$ 
2. How are the measurement properties of a daily diary impacted by creating a summary score from records with missing data. Specifically, reliability, validity, ability to detect change and group level and individual level interpretation thresholds.

3. How the ability to detect a known difference between two groups is impacted by creating a summary score from records with missing data.

The overall purpose of this work was to create a reliable online app which could be used by researchers and practitioners in the field to help them to decide missing data rules when planning to create summary scores in their research studies.

\section{Methods}

\section{Simulation of complete data}

Simulations were conducted in SAS version 9.4

Three different populations were simulated representing the differences in the day-to-day variability of different diseases. This included populations with low daily variability, moderate daily variability and high daily variability (Table 2) based on theoretical assumptions. An example of a condition which may have low variability is chronic pain, whereas a respiratory condition involving flares of symptoms may have high daily variability.

Table 2. Correlation structure for simulating daily variability

\begin{tabular}{|c|c|c|c|c|c|c|c|c|c|c|c|c|c|c|c|c|c|c|c|c|c|}
\hline & \multicolumn{7}{|c|}{ Low variability } & \multicolumn{7}{|c|}{ Moderate variability } & \multicolumn{7}{|c|}{ High variability } \\
\hline & 1 & 2 & 3 & 4 & 5 & 6 & 7 & 1 & 2 & 3 & 4 & 5 & 6 & 7 & 1 & 2 & 3 & 4 & 5 & 6 & 7 \\
\hline \multicolumn{22}{|l|}{1} \\
\hline 2 & .80 & & & & & & & .70 & & & & & & & .40 & & & & & & \\
\hline 3 & .70 & .80 & & & & & & .55 & .70 & & & & & & .25 & .40 & & & & & \\
\hline 4 & .60 & .70 & .80 & & & & & .40 & .55 & .70 & & & & & .10 & .25 & .40 & & & & \\
\hline 5 & .50 & .60 & .70 & .80 & & & & .25 & .40 & .55 & .70 & & & & .10 & .10 & .25 & .40 & & & \\
\hline 6 & .40 & .50 & .60 & .70 & .80 & & & .10 & .25 & .40 & .55 & .70 & & & .10 & .10 & .10 & .25 & .40 & & \\
\hline 7 & .30 & .40 & .50 & .60 & .70 & .80 & & .10 & .10 & .25 & .40 & .55 & .70 & & .10 & .10 & .10 & .10 & .25 & .40 & \\
\hline 8 & .00 & .00 & .00 & .00 & .00 & .00 & .30 & .00 & .00 & .00 & .00 & .00 & .00 & .30 & .00 & .00 & .00 & .00 & .00 & .00 & .30 \\
\hline $9+$ & .00 & .00 & .00 & .00 & .00 & .00 & .00 & .00 & .00 & .00 & .00 & .00 & .00 & .00 & .00 & .00 & .00 & .00 & .00 & .00 & .00 \\
\hline
\end{tabular}

*note - the correlation (0.30) between Day 7 and Day 8, and the correlation structure between Day 8 and Day 14 dictated the overall correlation between the two "weeks"

For each of the variability conditions, 1000 samples each with 400 records (representing participants) were simulated based on a normal distribution. Each participant had data simulated at two timepoints (Time 1 and Time 2) and each timepoint consisted of 7 days of data (datapoints). Participants were divided into treatment and control groups and scores were simulated to reflect a known treatment benefit at Time 2 (a group level of difference of around $~ 0.425-0.650$ points on an 11-point NRS scale). Data were simulated, and the known treatment benefits chosen, in such a way that known differences could be detected using between groups significance tests around $80 \%$ of the time given the sample size.

The simulated datapoints represented the underlying (and unobserved) latent scores for each participant, which are assumed to follow a normal distribution across the population. Latent scores are assumed to represent the true score of the patient without measurement error. As such, for Time 1, each of the 7 days of data was simulated on a continuous scale and had a mean of 5 and a standard deviation of 2 across the entire sample. The relationships between each day of data were governed by the correlation matrices displayed in Table 2 and were dependant on the variability condition. Although the correlation matrices in Table 2 represent the underlying intent behind the simulation, these exact matrices could not be used as they were not positive definite (a property required of correlation matrices). These matrices were transformed into positive definite matrices using the "nearPD" function in the R package "Matrix", which implements the algorithm detailed in Higham (2002) [17]. The resulting matrices were similar to those detailed in Table 2, but with near-zero elements rather than the zero elements displayed. 
For Time 2, latent scores for each group were derived as part of the same underlying correlation matrix governing the Time 1 simulation. A low correlation of 0.30 was specified between days that spanned Time 1 and Time 2 (i.e. between Day 7 of Time 1 and Day 1 of Time 2 ). The same correlation matrix as Time 1 (Table 2) was employed for the Time 2 relationships, however a normal distribution with a mean of 5 was used for the control group and a mean of between 4.350 and 4.575 was used for the treatment group. These values were chosen to allow for a chance of finding a between groups effect with a power of 0.8 using a two-sided test with an alpha value of 0.05 . To assess the relationship between the two timepoints, a correlation was performed between the mean of all latent datapoints at Time 1 and Time 2 . These values had a low, but consistent relationship in line with the manner in which these two timepoints were simulated $(r=0.1-0.2)$

Once the latent scores for Time 1 and Time 2 were developed, observed scores on a 0-10 numeric rating scale (NRS) were created by rounding the non-integer latent score for each timepoint to the nearest integer. This led to values between -1 and 13 , which were then restrained to lie between 0 and 10. A summary score was created for Time 1 and Time 2 as the mean of all 7 days of observed scores. This is referred to throughout as the Observed Summary Score and due to the rounding and truncation, introduced the "error" in measurement assumed when asking patients to complete a questionnaire.

Additional variables simulated as part of this analysis are detailed in supplementary material 1.

\section{Introduction of missing data}

Missing data was introduced into each scenario at Time 2 and followed the three missingness mechanisms (missing completely at random [MCAR], missing at random [MAR] and missing not at random [MNAR]).[10,12]

Missing data was introduced in increasing proportions. This means that either $10 \%, 20 \%, 30 \%$, or $40 \%$ of the sample were missing either 1 , 2 , 3 , $4,5,6$ or all 7 days of data. When all 7 days of data were missing for a proportion of the sample, this is akin to an analysis which only uses participants with complete data. In total (not including the full dataset arising from the initial simulation) this led to 28 scenarios for each of the 3 daily variability conditions for each of the missingness mechanisms, for a total of 252 scenarios involving some level of missing data.

The different missingness mechanisms were used to decide both on the participants selected to have datapoints deleted and the datapoints within participants' records that would be deleted. Participants selected to receive missing data were based on:

1. MCAR: Participants were selected completely at random

2. MAR: Participants were selected based on their Observed Summary Score at Time 1, with participants with a more severe response at Time 1 being more likely to be selected.

3. MNAR: Participants were selected based on their Observed Summary Score at Time 2.

Datapoints selected to receive missing data were based on:

1. MCAR: datapoints were selected completely at random

2. MAR: Datapoints were weighted for selection based on the severity of the previous day's severity. The higher the value of the previous days observed score, the more likely the datapoint would be selected for deletion. When the previous day was missing, the next available day was used. When there was no previous day, the Time 1 Observed Summary Score was used as a weight.

3. MNAR: datapoints were selected based on the severity of the observed datapoint for that day. The higher the value of that day's observed score, the more likely the datapoint would be selected for deletion.

For each participant in each scenario the Observed Summary Score for Time 2 was recalculated as the mean of all remaining datapoints. When all 7 days of data had been removed, no Observed Summary Score was created as there was no data from which to create a summary score.

These metrics were used in the assessment of the performance and psychometric properties of the Observed Summary Score under different missingness conditions.

\section{Assessment of Observed Summary Score Estimate, Psychometric properties and Efficacy Analysis Performance}

Prior to showing the impact on psychometric properties, the performance of the Observed Summary Score created from records with missing data was assessed in terms of bias and root mean squared error. Bias was assessed as the difference between the Observed Summary Score at Time 2 created from partial data and the "true" Observed Summary Score which was created from the complete data before missing data was added. This was calculated for each sample in each missingness scenario:

Page 5/10 
- Mean of (Participant T2 Observed Summary Score from partial data - Participant T2 Observed Summary Score from complete data)

In the following analyses, the psychometric performance of the Observed Summary Score created from the complete simulated data was compared to psychometric performance of the Observed Summary Score for each missingness scenario. This included assessments of convergent and known-groups methods validity, test-retest reliability, defining meaningful change thresholds at the individual and group level and, finally, the impact on both responder analysis and between groups differences. The description of these analyses is presented in supplementary material 2.

\section{Results}

Due to the complex design of this study, and the comprehensive range of scenarios that have been explored, a full assessment of the results is not possible in the confines of this publication. However, the results as they relate to a typical example will be displayed here, and the reader is encouraged to interact with the results themselves on the interactive tool, which contains all scenarios described within this work. The app can be obtained through contacting the corresponding author.

The results displayed here present a comparison of scenarios where $20 \%$ of a sample of participants with moderate daily disease variability have between 1 and 6 days of daily diary and the remaining $80 \%$ of the sample have complete data. The results are displayed in terms of how far the Observed Summary Score estimates and their respective measurement properties vary compared to: 1) complete data; and 2) a scenario where the $20 \%$ of participants with missing data are removed from the analysis (RFA; as is typically the case in practice when they do not have enough data to create a summary score). Here, MAR and MNAR will be reported as these are the most realistic missing data assumptions for clinical trial work. The results will be presented in terms of the median bias and the upper and lower interquartile range (IQR) of the estimates from the 1000 samples attributed to the respective missingness scenario.

\section{Observed Summary Score estimate}

Psychometric properties relating to the observed summary score should be considered in the context of the instrument and the range of possible scores that may be observed. Although data for this study are simulated, results can still be interpreted in terms of the range of possible scores (Observed daily measurements on a 0-10 integer scale, averaged over a week for Observed Summary Score) and the group level of change simulated over time (0.425 to 0.650$)$.

For MNAR data, Observed Summary Score estimates when $20 \%$ of moderate variability participants were RFA had a median bias of 0.12 , which in the context of this study accounts for between 18.5 and $28.2 \%$ of the simulated between groups difference of 0.425 to 0.650 . The lower IQR exceeded a reduction in Observed Score of 0.14 points, and the upper IQR of Observed Summary Score also indicated a reduction in score (0.09), indicating that the mean Observed Summary Score of most samples were biased away from the true simulated data. When participants with missing data were retained and an Observed Summary Score was created, a similar but attenuated bias was observed for all scenarios where participants had at least 3 days of daily diary data.

When the data were MAR, the Observed Summary Score estimate when participants with missing data were RFA showed minimal bias (median bias $=0.01$ ) and an IQR which spanned from -0.03 to 0.02 . For the scenarios where an Observed Summary Score was created despite participant records containing missing values, bias and IQR both increased with increasing missingness. When participants had at least 5 days of data the median Observed Summary Score was similar to the scenario when participants with missing data were RFA, although the IQR of scenario with 5 days of complete data was considerably smaller than for the RFA scenario. Furthermore, the medians and lower quartiles for scenarios with 4 days of daily diary data or more were closer to 0 than the RFA scenario, indicating that these scenarios were more likely to have estimates closer to the complete simulated Observed Summary Score. However, the upper quartiles of these scores did not include 0 . This means that despite being more proximal to the true, simulated Observed Summary Score, most samples in scenarios where participants were not RFA displayed a small but consistent bias. Figure 1 displays this as boxplots showing the overall pattern when participants are RFA is more accurate, but less precise than creating the Observed Summary Score from all available data.

\section{Psychometric Properties and Efficacy Analysis}

Alongside the overall summary score estimate, psychometric properties including validity, reliability and ability to detect change were also explored. These results are summarised in Table 3, although further details are provided in the supplementary material 3.

Table 3: Summary of results for further psychometric properties and efficacy analyses in the scenario where $20 \%$ of a sample of participants with moderate daily disease variability have between 1 and 6 days of data and the remaining $80 \%$ of the sample have complete data 


\begin{tabular}{|c|c|c|}
\hline Analysis & Summary & Recommendation \\
\hline $\begin{array}{l}\text { Convergent } \\
\text { Validity }\end{array}$ & $\begin{array}{l}\text { Cases with at least four days of data comparable to RFA. Bias minimal regardless of } \\
\text { approach. }\end{array}$ & $\begin{array}{l}\text { Use cases with at least four } \\
\text { days of data }\end{array}$ \\
\hline $\begin{array}{l}\text { Known } \\
\text { groups } \\
\text { validity }\end{array}$ & $\begin{array}{l}\text { Only including participants with three or more days of data generally limits percent effect size } \\
\text { bias to } 10 \% \text {. Removing incomplete cases completely results in a median value closer to true } \\
\text { underlying values of the sample but demonstrate a large amount of skew in the positive } \\
\text { direction (meaning spurious relationships were more likely to be observed). }\end{array}$ & $\begin{array}{l}\text { Use cases with at least } \\
\text { three days of data }\end{array}$ \\
\hline $\begin{array}{l}\text { Test-retest } \\
\text { reliability }\end{array}$ & $\begin{array}{l}\text { Median change in ICC less than } 0.05 \text { for all cases (including when participants with missing } \\
\text { data are RFA) }\end{array}$ & $\begin{array}{l}\text { Use cases with at least } \\
\text { three days of data }\end{array}$ \\
\hline $\begin{array}{l}\text { Ability to } \\
\text { detect } \\
\text { change }\end{array}$ & $\begin{array}{l}\text { Scenarios where participants were RFA demonstrated relatively precise and accurate ability to } \\
\text { detect change. More negative skew observed as fewer data points were available, suggesting } \\
\text { a reduced ability of the observed summary score to be able to detect a change where a } \\
\text { change exists. }\end{array}$ & $\begin{array}{l}\text { Use cases with at least five } \\
\text { days of data }\end{array}$ \\
\hline $\begin{array}{l}\text { Minimally } \\
\text { Important } \\
\text { Difference } \\
\text { Derivation }\end{array}$ & $\begin{array}{l}\text { Scenarios where participants were RFA showed greater bias than scenarios where } \\
\text { participants with at least two days of diary data were included. Variability larger when using } \\
\text { participants with one or two days of data in comparison to RFA. }\end{array}$ & $\begin{array}{l}\text { Use all observed data to } \\
\text { create the summary score, } \\
\text { provided most participants } \\
\text { have at least three days of } \\
\text { data. }\end{array}$ \\
\hline $\begin{array}{l}\text { Responder } \\
\text { Definition } \\
\text { Derivation }\end{array}$ & $\begin{array}{l}\text { Similar results to MID derivation when using lower } \mathrm{Cl} \text { of minimal change group to derive RD. } \\
\text { When using ROC, RFA demonstrated no bias (likely due to methods returning a categorical cut } \\
\text { point for each sample rather than a continuous score, limiting variability). }\end{array}$ & $\begin{array}{l}\text { Use cases with at least } \\
\text { three days of data. }\end{array}$ \\
\hline $\begin{array}{l}\text { Between } \\
\text { groups } \\
\text { differences }\end{array}$ & $\begin{array}{l}\text { Reduction in the number of samples which reported statistically significant between group } \\
\text { differences when participants were RFA. }\end{array}$ & $\begin{array}{l}\text { Use cases with at least } \\
\text { three days of data* }\end{array}$ \\
\hline \multirow[t]{2}{*}{$\begin{array}{l}\text { Responder } \\
\text { Analyses }\end{array}$} & $\begin{array}{l}\text { When participants with missing data were RFA, there was a very large reduction in the number } \\
\text { of responders in the trial compared to complete data. When using any available data, the } \\
\text { number of responders artificially increased regardless of the specific scenario. }\end{array}$ & $\begin{array}{l}\text { Use only complete cases to } \\
\text { avoid artificially inflating } \\
\text { treatment effect }\end{array}$ \\
\hline & & $\begin{array}{l}\text { Use of cases with at least } 5 \\
\text { days of data may be } \\
\text { appropriate as positive bias } \\
\text { is limited. }\end{array}$ \\
\hline
\end{tabular}

*potentially fewer days could be accepted according to this data. The clause of three days of data is employed because the false positive rate has not been assessed in this study. However, previous work has shown that this does not increase when 3-days of data are used to make a summary score [15, Floden in preparation]

\section{Discussion}

This study aimed to develop a resource for identifying the circumstances under which participants with missing daily diary data should be removed from an analysis compared to when they should be included, despite having incomplete data. The complete tool is now available for general use. Taking a single possible scenario, this study has shown some interesting results regarding the impact that missing data handling decisions have on the psychometric properties, interpretation and ultimately, results of a daily diary study.

This work has shown that the impact of missing data handling strategies varies depending on the analysis being conducted. For the majority of the measurement property assessments in the explored scenario, it was apparent that existing rules of thumb (creating a summary score for participants with 4 or more observed days of data) are comparable, if not preferable to removing these participants from the analysis. Despite the bias in the psychometric parameters (especially when the data were explicitly MNAR), the precision of the estimates made them likely more reliable than when removing participants from the analysis.

However, when it comes to deriving a meaningful change threshold, the results were more nuanced. Group-level minimal important difference thresholds, the point at which the difference between two treatment arms is meaningful, are likely more accurately defined by using all available data to create the summary score, at least when the data are MAR. When defining individual-level responder definitions, however, it appears that removing participants from the analysis is the best way to avoid a negative skew in the data. A negative skew in the data could lead to a smaller-than-appropriate responder definition, the consequence of which could be that participants are incorrectly classified as responders. The conservative approach to avoid this scenario from occurring is to remove participants with missing data from the analysis prior to deriving a responder definition. 
When it comes to interpreting the results of a trial which creates a summary score from daily diary data the results are a lot more difficult to practically interpret. On the one hand, there is a clear trend in support of retaining data. The results showed a clear under-estimate of the efficacy effect at both a group and individual level when participants with missing data were RFA rather than retained with a summary score created from available data. This is also supported by theory, as it stands that a reduction in participant numbers leads to a reduction in power to detect a difference where one exists. However, as shown by the responder analysis results, creating a summary score from all available data arbitrarily increases the number of participants who are classed as responders, thus potentially leading to results where a treatment effect may be supported by results, but does not in reality exist.

Overall, the way the measurement properties of a summary score are assessed, interpreted and analysed in terms of efficacy should relate to the endpoint employed for the study. This does mean, however, that researchers should build their endpoint hierarchies with the knowledge that the chosen method to construct the summary score will impact their results, deviating in some way from the unobservable truth. Given the recent shift towards the estimand language[20], researchers should make sure that their objectives clearly indicate the construct they are estimating with their summary scores. The tool developed as part of this study allows researchers to under the effect that their chosen method of summary score creation will have on their results, and ultimately consider robust derivation rules and alternative summary score derivation rules to be used as a sensitivity analysis in the case that the data may be MNAR.

To conclude, the current work suggests that the way a summary score is derived with regards to missing data has an impact on the score's measurement properties, interpretation and ability to detect an efficacy effect. However, the way the summary score is derived does not have a uniform impact across these analyses. Furthermore, as the tool created as part of this work shows, this relationship is further moderated by the daily variability inherent in the condition, the number of participants with missing data and the missingness mechanism underlying the incomplete data. The creation of summary scores is a form of data reduction. Specifically in the case of averaging over missing data, this is a form of imputation. As such, perhaps other methods of handling daily diary data are necessary, such as intensive longitudinal models where each daily diary observation can be, for example, nested within each week. Although this work allows insight into how to conduct research under the present paradigm of creating summary scores, it is perhaps instead time to start exploring methods which can model change across time using each individual datapoint available.

\section{Declarations}

Funding: This work was not funded, but was conducted while the authors were employees of Adelphi Values, UK.

Conflicts of interest/Competing interests: None

Availability of data and material (data transparency): Data underlying the simulation and the online tool is available from the corresponding author on request

Code availability (software application or custom code): If code is required, requests can be made to the corresponding author and will be considered on a case for case basis.

Authors' contributions: P.G. conceived of the presented idea. P.G. and A.W. developed the theory and performed the computations. P.G. and A.W. verified the analytical methods. A.W. built the online tool. E.B. supervised the findings of this work. All authors discussed the results and contributed to the final manuscript

Ethics approval: Not required

Consent to participate: Not required

Consent for publication: Not required

\section{References}

1. Okupa, A. Y., Sorkness, C. A., Mauger, D. T., Jackson, D. J., \& Lemanske Jr, R. F. (2013). Daily diaries vs retrospective questionnaires to assess asthma control and therapeutic responses in asthma clinical trials: is participant burden worth the effort?. Chest, 143(4), 993-999.

2. Lewandowski, A. S., Palermo, T. M., Kirchner, H. L., \& Drotar, D. (2009). Comparing diary and retrospective reports of pain and activity restriction in children and adolescents with chronic pain conditions. The Clinical journal of pain, 25(4), 299.

3. Bennett, A. V., Amtmann, D., Diehr, P., \& Patrick, D. L. (2012). Comparison of 7-day recall and daily diary reports of COPD symptoms and impacts. Value in Health, 15(3), 466-474.

4. Coons, S. J., Eremenco, S., Lundy, J. J., O’Donohoe, P., O’Gorman, H., \& Malizia, W. (2015). Capturing patient-reported outcome (PRO) data electronically: the past, present, and promise of ePRO measurement in clinical trials. The Patient-Patient-Centered Outcomes Research, 8(4),

Page $8 / 10$ 
301-309.

5. Stone, A. A., Shiffman, S., Schwartz, J. E., Broderick, J. E., \& Hufford, M. R. (2002). Patient non-compliance with paper diaries. Bmj, 324(7347), 1193-1194.

6. Tiplady, B. (2010). Diary Design Considerations. EPro: Electronic Solutions for Patient-reported Data, 167.

7. Bingham, C. O., Gaich, C. L., DeLozier, A. M., Engstrom, K. D., Naegeli, A. N., De Bono, S., ... \& Taylor, P. C. (2019). Use of daily electronic patient-reported outcome (PRO) diaries in randomized controlled trials for rheumatoid arthritis: rationale and implementation. Trials, 20(1), 1-8.

8. Strunk, R. C., Bender, B., Young, D. A., Sagel, S., Glynn, E., Caesar, M., \& Lawhon, C. (2002). Predictors of protocol adherence in a pediatric asthma clinical trial. Journal of allergy and clinical immunology, 110(4), 596-602.

9. Holzbaur, E., \& Ross, J. (2014). Risks, impacts, and mitigation of missing epro data on clinical trials. Value in Health, $17(3)$, A204.

10. Fairclough, D. L. (2010). Design and analysis of quality of life studies in clinical trials. CRC press.

11. Coens, C., Pe, M., Dueck, A. C., Sloan, J., Basch, E., Calvert, M., ... \& Bottomley, A. (2020). International standards for the analysis of qualityof-life and patient-reported outcome endpoints in cancer randomised controlled trials: recommendations of the SISAQOL Consortium. The Lancet Oncology, 21(2), e83-e96.

12. Bell, M. L., \& Fairclough, D. L. (2014). Practical and statistical issues in missing data for longitudinal patient-reported outcomes. Statistical methods in medical research, 23(5), 440-459.

13. Bolger, N., Davis, A., \& Rafaeli, E. (2003). Diary methods: Capturing life as it is lived. Annual review of psychology, 54(1), 579-616.

14. Griffiths, P., Floden, L. L., \& Hudgens, S. (2017, October). Scoring and interpretation of daily diary data in the presence of non-ignorable missing data. In QUALITY OF LIFE RESEARCH (Vol. 26, No. 1, pp. 70-70). VAN GODEWIJCKSTRAAT 30, 3311 GZ DORDRECHT, NETHERLANDS: SPRINGERGriffiths, P., Floden, L. L., \& Hudgens, S. (2017, October). Scoring and interpretation of daily diary data in the presence of non-ignorable missing data. In QUALITY OF LIFE RESEARCH (Vol. 26, No. 1, pp. 70-70). VAN GODEWIJCKSTRAAT 30, 3311 GZ DORDRECHT, NETHERLANDS: SPRINGER.

15. Griffiths, P., Floden, L., Doll, H., Morris, M., \& Hudgens, S. (2018, October). Psychometric properties in the face of missing data-a simulation study assessing the effect of missing data on test-retest reliability in diary studies. In QUALITY OF LIFE RESEARCH (Vol. 27, pp. S55-S55). VAN GODEWIJCKSTRAAT 30, 3311 GZ DORDRECHT, NETHERLANDS: SPRINGER.

16. Fayers, P. M., \& Machin, D. (2013). Quality of life: the assessment, analysis and interpretation of patient-reported outcomes. John Wiley \& Sons.

17. Higham, N. J. (2002). Computing the nearest correlation matrix-a problem from finance. IMA journal of Numerical Analysis, 22(3), 329343.

18. Norman, G. R., Stratford, P., \& Regehr, G. (1997). Methodological problems in the retrospective computation of responsiveness to change: the lesson of Cronbach. Journal of clinical epidemiology, 50(8), 869-879.

19. Terluin, B., Eekhout, I., \& Terwee, C. B. (2017). The anchor-based minimal important change, based on receiver operating characteristic analysis or predictive modeling, may need to be adjusted for the proportion of improved patients. Journal of clinical epidemiology, 83 , 90 100.

20. Lawrance, R., Degtyarev, E., Griffiths, P., Trask, P., Lau, H., D’Alessio, D., ... \& Rufibach, K. (2020). What is an estimand \& how does it relate to quantifying the effect of treatment on patient-reported quality of life outcomes in clinical trials?. Journal of Patient-Reported Outcomes, $4(1), 1-8$.

\section{Figures}




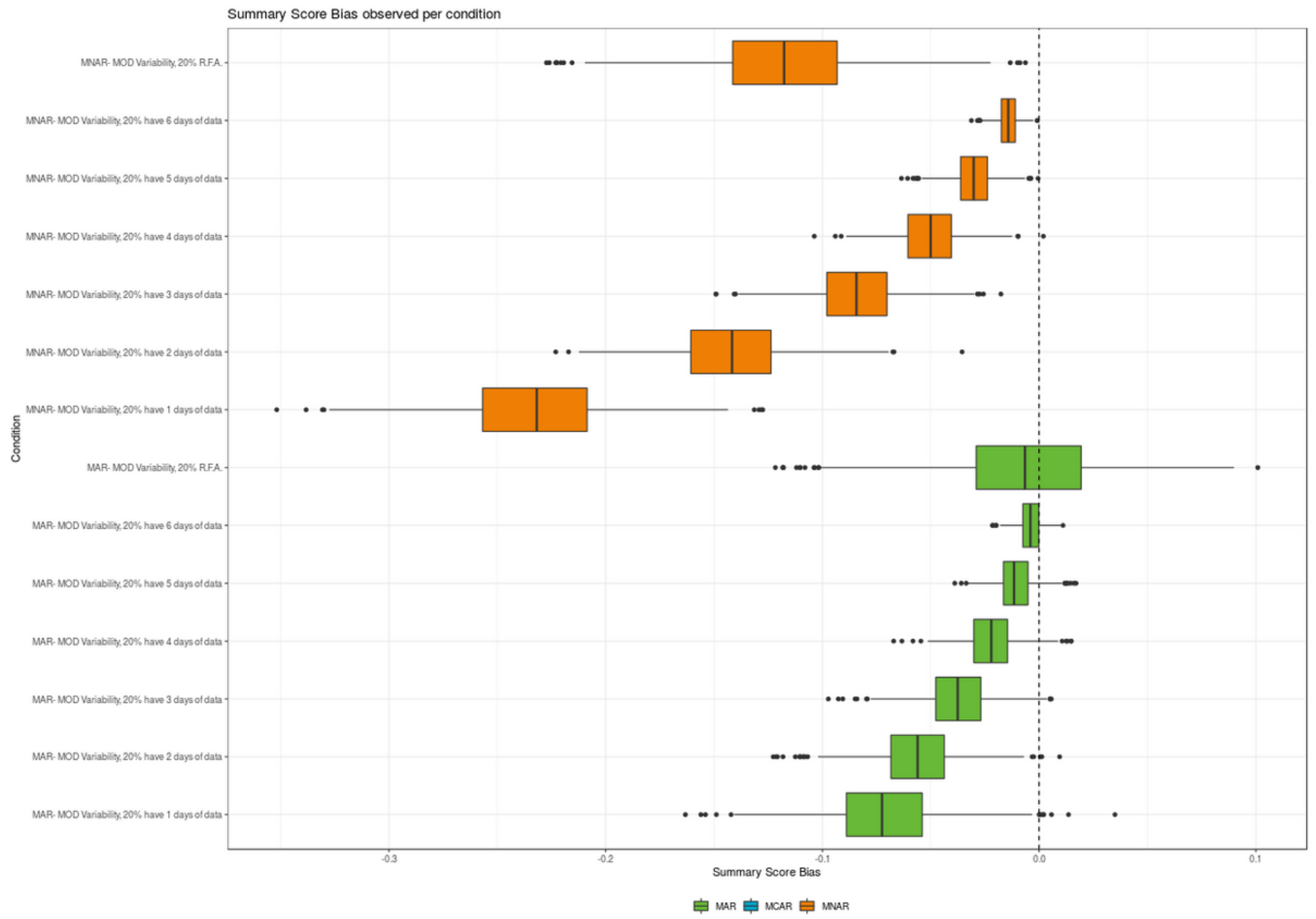

Figure 1

Observed Summary Score Median and IQR. Deviation from dashed line represents deviation from complete data

\section{Supplementary Files}

This is a list of supplementary files associated with this preprint. Click to download.

- SupplementaryMaterial.pdf 